\title{
Engineering Specificity Changes on a RanBP2 Zinc Finger that Binds Single-Stranded RNA**
}

\author{
Marylène Vandevenne, Mitchell R. O'Connell, Stephanie Helder, Nicholas E. Shepherd, \\ Jacqueline M. Matthews, Ann H. Kwan, David J. Segal, and Joel P. Mackay*
}

\begin{abstract}
The realization that gene transcription is much more pervasive than previously thought and that many diverse RNA species exist in simple as well as complex organisms has triggered efforts to develop functionalized RNA-binding proteins (RBPs) that have the ability to probe and manipulate RNA function. Previously, we showed that the RanBP2-type zinc finger $(Z F)$ domain is a good candidate for an addressable single-stranded-RNA (ssRNA) binding domain that can recognize ssRNA in a modular and specific manner. In the present study, we successfully engineered a sequence specificity change onto this $Z F$ scaffold by using a combinatorial approach based on phage display. This work constitutes a foundation from which a set of RanBP2 ZFs might be developed that is able to recognize any given $R N A$ sequence.
\end{abstract}

O ur appreciation of the diversity of RNA biology has grown dramatically over the last ten years. In particular, the advent of deep-sequencing technologies has shown that a plethora of RNA species that do not code for proteins are found in the cells of complex organisms. ${ }^{[1,2]}$ It has also become clear that the messenger RNAs of essentially all human proteins undergo alternative splicing, and therefore can code for several protein products that might display distinct functional properties. ${ }^{[3]}$ Together, these RNA-centered processes have the potential to exert powerful effects in the cell. Indeed, it has already been shown that approximately $15 \%$ of all diseases arise from problems with mRNA processing. ${ }^{[4]}$ For example, specific mutations in the dystrophin gene cause Duchenne muscular dystrophy by impairing the maturation of

[*] Dr. M. Vandevenne, Dr. M. R. O'Connell, ${ }^{[+]}$S. Helder,

Dr. N. E. Shepherd, Prof. J. M. Matthews, Dr. A. H. Kwan,

Prof. J. P. Mackay

School of Molecular Bioscience, University of Sydney

Sydney, N.S.W 2006 (Australia)

E-mail: joel.mackay@sydney.edu.au

Prof. D. J. Segal

Genome Center and Department of Biochemistry and Molecular Medicine, University of California Davis

Davis, CA 95616 (USA)

$\left.{ }^{+}\right]$Current Address: Department of Molecular and Cell Biology University of California, Berkeley

Berkeley, CA 94609 (USA)

[***] This work was supported by the National Health and Medical Research Council, Australia. We thank Prof. Matteo Pellegrini and Marco Morselli from UCLA for their assistance and help in the deepsequencing experiment, and Dr. Artem Zykovich and Abigail Yu for providing the software MERMADE and for assistance with its use. Supporting information for this article is available on the WWW under http://dx.doi.org/10.1002/anie.201402980. the pre-mRNA. ${ }^{[5]}$ Similarly, nonsense mutations in BRCA $1^{[6]}$ and fibrillin $1^{[7]}$ cause inappropriate exon skipping and are associated with breast cancer and Marfan syndrome, respectively. Global deregulation of $3^{\prime}$-UTR processing has also been observed very recently in cancer, ${ }^{[8]}$ cardiac hypertrophy, ${ }^{[9]}$ and a range of other disorders (reviewed in Ref. [8]). RNA is also intimately involved in infectious disease. For example, all retroviral infections require the injection of the retroviral single-stranded RNA genome into the host cell. ${ }^{[10]}$

Currently, our understanding of the functions of these many thousands of RNA species is in its infancy. RNAbinding proteins with addressable specificity would, therefore, be of great value in the analysis and manipulation of cellular RNAs. Some success has already been reported with Pumilio (PUF) repeat proteins, for which a complete recognition code has been established. ${ }^{[11,12]}$ Engineered PUF domains have been combined with various effector domains to create designer splicing factors, site-specific RNA endonucleases, and RNA probes. ${ }^{[12,13]}$ However, despite the apparent simplicity of the two amino acid recognition code, a number of complications exist. For example, different RNA binding modes have been observed, thus complicating the prediction and control of sequence specificity. ${ }^{[14]}$ In addition, recent studies have shown that PUF domains can interact with $\mathrm{CPEB}^{[15]}$ and Ago proteins, ${ }^{[16]}$ which can also lead to an alteration of their sequence specificity. ${ }^{[17]}$ Finally, their highly repetitive nature and/or intrinsic physiochemistry of PUF proteins make them relatively difficult to manipulate.

Previously, we demonstrated that the RanBP2-type zinc finger (ZF) offers promise as an alternative scaffold on which to build designer single-stranded RNA-binding proteins (ssRBPs). ${ }^{[18]}$ This modular $\mathrm{ZF}$ recognizes ssRNA in a sequence-specific manner, recognizing three nucleotides of ssRNA ${ }^{[19]}$ We assembled a tandem array of three ZFs that could recognize a specific nine-nucleotide sequence with high affinity and specificity. ${ }^{[18]}$ In principle, a set of 64 variant ZFs could be designed to recognize all trinucleotide sequences, thereby providing an addressable RNA-binding platform analogous to the well-described DNA-binding ZF technology. ${ }^{[20]}$ We demonstrate herein that combinatorial selection can be used to derive a $\mathrm{ZF}$ variant with altered sequence specificity, thereby providing proof-of-principle that RanBP2 ZFs can serve as such a platform.

Our published structural (Figure 1 A) and biochemical data show that each of the two RanBP2 ZFs from the human protein ZRANB2 (F1 and F2) binds ssRNA with a preference for the trinucleotide GGU. ${ }^{[19,21]}$ Similarly, the RanBP2-type ZF from RBM5 binds most tightly to GGG. ${ }^{[21,22]}$ In an effort to find sequence variants that recognize different trinucleo- 
A)

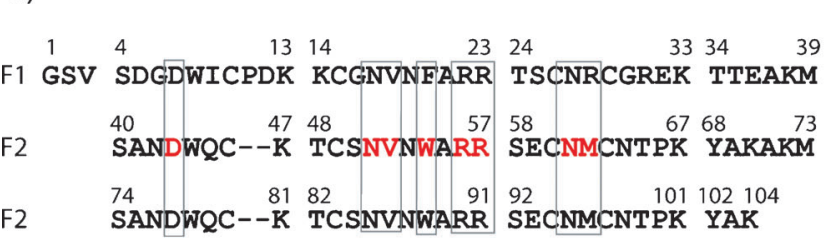

C)

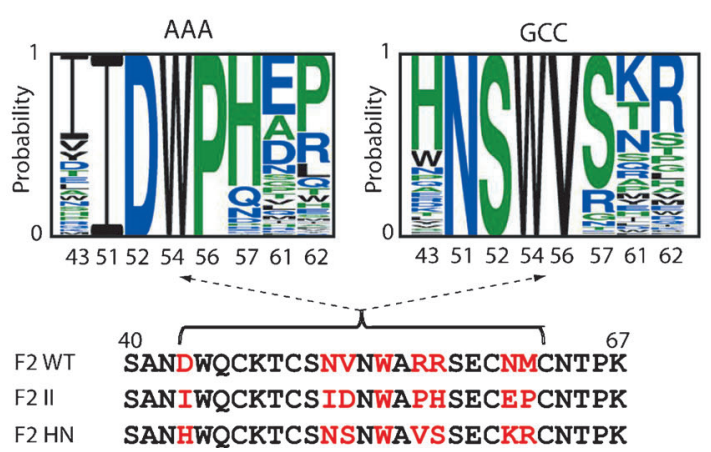

Figure 1. A) Representation of the experimental setup used to select RanBP2 ZF variants with altered specificity, in which phage particles display members of the F1-F2 mut $^{-F 2}$ library on their surface. The structure of ZRANB2-F2 in complex with its target GGU sequence is shown. RNA-binding residues that were randomized in the library are shown in red. B) Amino acid sequence of the WT three-ZF construct used for phage display, RNA binding residues are highlighted in gray boxes and shown in red in the middle F2. C) Web logos indicating the most dominant consensus sequences enriched during the selection of the different target RNA sequences. Each motif was generated using the program MERMADE ${ }^{[24]}$ from a subset of enriched sequences from the final round of selection (see the Supporting Information). Each amino acid present in these motifs represents one of the eight randomized residues of the middle F2 (note that they are not contiguous in the sequence-numbers are shown below the motif). The $F 2_{W T}$ sequence and the sequences of the $F 2_{H N}$ and $F 2_{\| I}$ variants are also shown. The randomized binding residues are highlighted in red.

tides, we created a library of about $3 \times 10^{8}$ variants of ZRANB2-F2, in which eight residues that make direct contact with the RNA in the F2-RNA crystal structure ${ }^{[23]}$ were randomized as follows: residues D43, N51, V52, R56, R57, N61, and M62 (numbering is detailed in Figure 1B) were permitted to be any residue except for glycine and cysteine. W54, which stacks between the two guanine bases, was only allowed to be a hydrophobic residue (W, Y, F, I, H, and L) in an effort to maintain a hydrophobic interaction with the bases. The F2 variant library was created in the context of a three-ZF construct in which the variant $\mathrm{ZF}$ was flanked by
F1 and a wild-type (WT) copy of F2 (creating F1-F2 ${ }_{\text {mut }}-\mathrm{F} 2$ ) and the whole construct was cloned into the vector pComb3XSS (provided by the Barbas research group). Following incorporation into the genome of filamentous M13 bacteriophage, polypeptides encoded by this library were displayed on the surface of the phage in fusion with protein $\mathrm{pIII}$ and used in a standard phage display panning and selection procedure. We carried out experiments to select either for variants that preferentially bound GCC or for variants that bound AAA, by using baits comprising the ninenucleotide ssRNA sequences GGUGCCGGU or GGUAAAGGU, respectively. In each case, the RNA was linked to biotin and immobilized on streptavidin-coated magnetic beads. The flanking F1 and F2 ZFs and the flanking GGU sequences were included to provide register for the variant $\mathrm{ZF}\left(\mathrm{F} 2_{\text {mut }}\right)$ and to enhance binding. During the selection process, mixtures of ssRNAs with the sequence GGUNNNGGU (where $\mathrm{N}$ is any nucleotide) were included to provide negative selection pressure.

Six rounds of selection and amplification were carried out. The $F 2_{\text {mut }}$ portion of the library for each selection was amplified by PCR and subjected to deep sequencing (Illumina Hiseq 2500) following each round of panning. Analysis of the pooled sequences using the program MERMADE ${ }^{[24]}$ (see the Supporting Information) revealed strong enrichment of specific variants in the later selection rounds. This strong decrease in sequence diversity and the enrichment of particular variants in the later rounds suggested that selection had taken place (see Figure S1 in the Supporting Information). Figure $1 \mathrm{C}$ shows the most dominant sequence motif for each selection and a comparison of typical sequences with the WT F2 sequence. Besides the two most dominant variants, we chose a subset of four additional variants that were also strongly enriched in the later selection rounds for further analysis (their amino acid sequence is detailed in Figure S2 in the Supporting Information). We expressed and purified the corresponding $\mathrm{F} 1-\mathrm{F} 2_{\text {mut }}-\mathrm{F} 2$ proteins and used microscale thermophoresis (MST) ${ }^{[25]}$ as a first screen to assess their RNA-binding properties (variants are named by the first two of the eight selected residues; Figure $2 \mathrm{~A}$ ).

The $\mathrm{F} 1-\mathrm{F} 2_{\mathrm{YT}}-\mathrm{F} 2$ and $\mathrm{F} 1-\mathrm{F} 2_{\mathrm{HN}}-\mathrm{F} 2$ variants bound more tightly to the sequence against which they were selected than they did to the "parent" sequence GGUGGUGGU (Figure $2 \mathrm{~A}$ ). We also tested the binding of the $\mathrm{F} 1-\mathrm{F} 2_{\mathrm{YT}} \mathrm{F} 2$ and $\mathrm{F} 1-$ $\mathrm{F} 2_{\mathrm{HN}}-\mathrm{F} 2$ variants against a different RNA (GUU and AAA, respectively) to further assess specificity. Although F1-F2 YT $^{-}$ F2 showed tighter binding to its AAA sequence than to GGU $\left(72 \pm 4\right.$ vs $\left.39 \pm 2 \times 10^{6} \mathrm{M}^{-1}\right)$, it showed a similarly high affinity for GUU $\left(71 \pm 6 \times 10^{6} \mathrm{M}^{-1}\right)$ and so was discounted from further analysis. In contrast, the $\mathrm{F} 1-\mathrm{F} 2_{\mathrm{HN}}-\mathrm{F} 2$ variant bound more tightly to its cognate target (GCC) than to either GGU or AAA $\left(9 \pm 1\right.$ versus $5.2 \pm 0.2$ and $2.8 \pm 0.2 \times 10^{6} \mathrm{M}^{-1}$, respectively). The difference was small but reproducible and warranted further attention. Unexpectedly, the F1-F2 ${ }_{\mathrm{VA}}-\mathrm{F} 2$ (Figure 2A) variant had a significantly higher affinity for GGU than did wild-type F1-F2 ${ }_{\mathrm{wT}}-\mathrm{F} 2(100 \pm 25$ versus $25 \pm 1 \times$ $\left.10^{6} \mathrm{M}^{-1}\right)$; however, the affinity of $\mathrm{F} 1-\mathrm{F} 2 \mathrm{VA}_{\mathrm{A}}-\mathrm{F} 2$ for its intended site AAA sequence was not as strong $\left(50 \pm 4 \times 10^{6} \mathrm{M}^{-1}\right)$, and so this variant was not pursued. 
A)

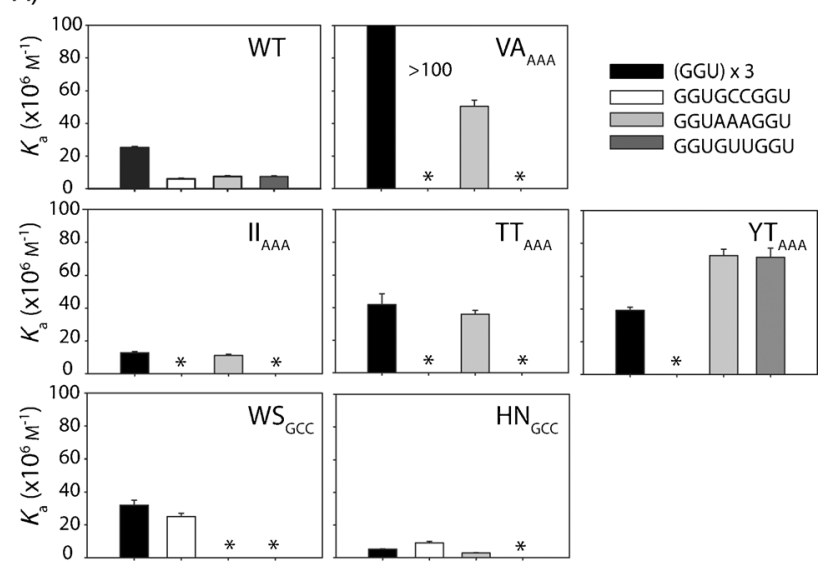

B)

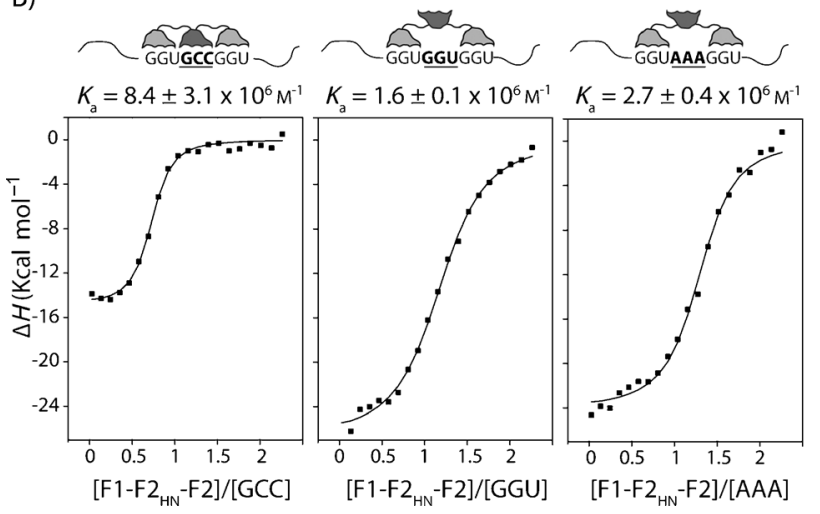

Figure 2. A) Binding affinities of the $W T$ and selected three-ZF variants $\mathrm{F} 1-\mathrm{F} 2_{\text {mut }}-\mathrm{F} 2$ with several RNA sequences. The binding affinities were measured using MST. The data points and bars represent, respectively, the means and propagated standard errors for the fits for two independent titrations; asterisks indicate that the binding affinities were not measured. Variants are named after the first two of the eight selected residues, and the target RNA that they were selected for is specified by the lower case 3-nucleotide sequence. B) ITC data for the titration of $\mathrm{F} 1-\mathrm{F}_{2} \mathrm{HN}^{-} \mathrm{F} 2$ to several indicated RNA sequences. The curves represent the integrated enthalpy changes per mol of injected protein. The fitted affinities are specified on the graphs and correspond to the mean and the propagated standard errors calculated from 2-3 independent titrations.

We also noted that the binding curve for the $\mathrm{F} 1-\mathrm{F} 2_{\mathrm{HN}^{-}}$ F2:GCC interaction showed two distinct binding events: the first $\left(K_{\mathrm{a}}=9 \pm 1 \times 10^{6} \mathrm{M}^{-1}\right)$ characterized by a positive thermophoresis and the second $\left(K_{\mathrm{a}}=2.6 \pm 0.1 \times 10^{6} \mathrm{M}^{-1}\right)$ exhibiting a negative thermophoresis (see Figure S3 in the Supporting Information). It is likely that the first binding event corresponds to the binding of the protein to its specific target site, whereas the second represents binding of the protein to either surrounding bases or to the RNA backbone. To corroborate the order of affinities observed for $\mathrm{F} 1-\mathrm{F} 2_{\mathrm{HN}}-\mathrm{F} 2$ by MST, we used isothermal titration calorimetry (ITC, Figure 2B).

The affinity measured for the $\mathrm{F} 1-\mathrm{F} 2_{\mathrm{HN}}-\mathrm{F} 2$ : GCC interaction is $8.4 \pm 3.1 \times 10^{6} \mathrm{M}^{-1}$, in close agreement with the affinity determined for the tighter MST binding event. The affinities for GGU and AAA are both substantially lower: $1.6 \pm 0.1 \times$ $10^{6} \mathrm{M}^{-1}$ and $2.7 \pm 0.4 \times 10^{6} \mathrm{M}^{-1}$, respectively. Taken together,
A)

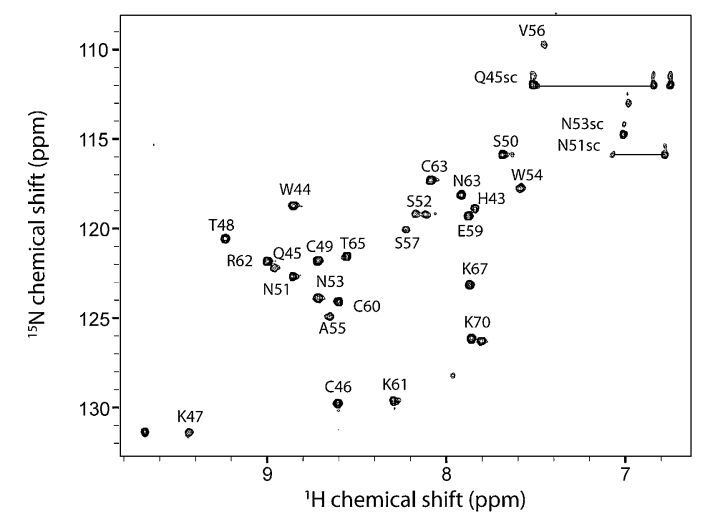

B)
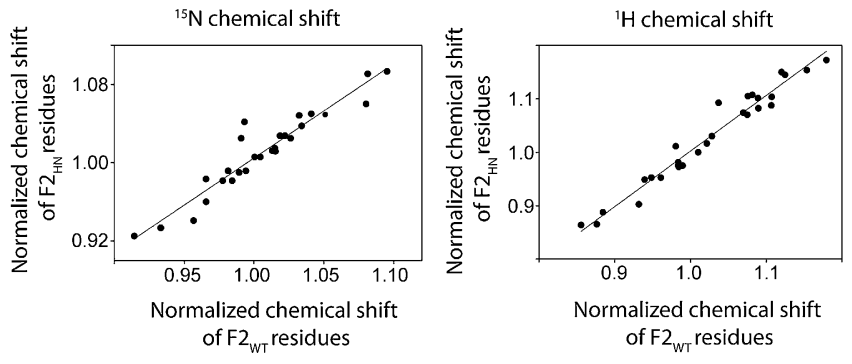

Figure 3. A) Assigned ${ }^{15} \mathrm{~N}-\mathrm{HSQC}$ spectrum of $\mathrm{F}_{\mathrm{HN}}$. The spectrum was recorded at $25^{\circ} \mathrm{C}$ in $10 \mathrm{~mm} \mathrm{Na}_{2} \mathrm{HPO}_{4}(\mathrm{pH} 7.0), 50 \mathrm{~mm} \mathrm{NaCl}, 1 \mathrm{~mm}$ dithiothreitol (DTT), $5 \%(\mathrm{v} / \mathrm{v}) \mathrm{D}_{2} \mathrm{O}$, and $2 \mu \mathrm{M}$ 4,4-dimethyl-4-silapentane-1-sulfonic acid (DSS). Cross-peaks are labeled with residue numbers from 43 to 70 , which correspond to the three-ZF construct numbering detailed in Figure 1. Side-chain (sc) resonances of asparagine and glutamine residues are indicated by a horizontal line. B) Plot of the chemical shifts of the corresponding backbone ${ }^{1} \mathrm{H}$ and ${ }^{15} \mathrm{~N}$ nuclei from $\mathrm{F} 2_{\mathrm{HN}}$ and the parent domain $\mathrm{F} 2$. To visualize the data, chemical shifts have been normalized so that the mean ${ }^{1} \mathrm{H}$ and ${ }^{15} \mathrm{~N}$ chemical shifts for $\mathrm{F} 2$ are 1.0 .

these data indicate that the selected $\mathrm{F}_{\mathrm{HN}}$ variant in the context of $\mathrm{F} 1-\mathrm{F} 2_{\mathrm{HN}}-\mathrm{F} 2$ can discriminate its target RNA sequence over the other related sequences. It is notable that the three- to fivefold sequence-discrimination ability of this variant is comparable to that observed for the WT protein F1$\mathrm{F} 2{ }_{\mathrm{WT}} \mathrm{F} 2$ (Figure $2 \mathrm{~A}$ ), even though the overall binding affinity is weaker for the selected variant; the measured binding affinities of the WT protein for GGU, GCC, and AAA are $25 \pm 0.5,5.9 \pm 0.3$, and $7.4 \pm 0.4 \times 10^{6} \mathrm{M}^{-1}$; respectively.

To probe the structure and mode of RNA binding of $\mathrm{F} 2 \mathrm{HN}$ more directly, we first expressed and purified uniformly ${ }^{15} \mathrm{~N}$ labeled $\mathrm{F}_{2} \mathrm{HN}$ and made backbone and partial side-chain assignments using ${ }^{15} \mathrm{~N}$-edited TOCSY and NOESY spectroscopy. The ${ }^{15} \mathrm{~N}$-HSQC spectrum of the domain (Figure $3 \mathrm{~A}$ ) displayed narrow line widths and good chemical shift dispersion, consistent with a well-ordered fold. Plots of the chemical shifts of the corresponding backbone $\mathrm{H}^{\mathrm{N}}, \mathrm{H}^{\alpha}$, and ${ }^{15} \mathrm{~N}$ nuclei from $\mathrm{F} 2_{\mathrm{HN}}$ and the parent domain $\mathrm{F} 2{ }_{\mathrm{WT}}$ (Figure $3 \mathrm{~B}$ ) show very strong linear correlations, with slopes near unity, thus indicating that $\mathrm{F}_{2} \mathrm{HN}$ takes up a fold that closely resembles that of $\mathrm{F} 2_{\mathrm{WT}}$. This observation is consistent with our idea that the RANBP2-type $\mathrm{ZF}$ is very tolerant of mutagenesis, an important property for a design scaffold. We next recorded the ${ }^{15} \mathrm{~N}$-HSQC spectra of $200 \mu \mathrm{M} \mathrm{F} 2_{\mathrm{HN}}$ in the 
presence of up to 1.9 molar equivalents of a ssRNA oligonucleotide with the sequence AAAGCCAAA. However, no chemical shift changes or changes in signal intensity were observed, even at the highest concentration of RNA (data not shown), which suggests that the association constant for the formation of any $\mathrm{F}_{\mathrm{HN}_{\mathrm{HN}}}$ RNA complex must be less than $1 \times$ $10^{2} \mathrm{M}^{-1}$. Consistent with this conclusion, we were also unable to detect a clear interaction between $\mathrm{F}_{2} \mathrm{HN}$ and RNA by MST.

Next, ${ }^{15} \mathrm{~N}$-HSQC spectra were recorded of ${ }^{15} \mathrm{~N}$-labeled F1$\mathrm{F} 2{ }_{\mathrm{HN}}-\mathrm{F} 2$ following the addition of increasing quantities of a ssRNA with the sequence GGUGCCGGU. The intensities of a specific subset of signals from residues in $\mathrm{F} 2{ }_{\mathrm{HN}}$ decreased more dramatically than the rest upon addition of the RNA (Figure 4); the observation of reductions in intensity rather than changes in chemical shift indicates that the interaction is, overall, in slow exchange on the chemical shift timescale (consistent with the measured affinity). In the flanking F1 and F2 ZFs, the identity of these residues agrees generally with published data for the F2-RNA interaction. ${ }^{[19]}$ The amplitude and direction of the changes observed for $\mathrm{F} 2_{\mathrm{HN}}$ are similar to those observed for $\mathrm{F} 1$, which suggests that $\mathrm{F} 2_{\mathrm{HN}}$ makes comparable interactions with its target site.

How can the data on the lone $\mathrm{F} 2{ }_{\mathrm{HN}}$ versus the same domain in a $3-\mathrm{ZF}$ protein be reconciled? That is, how is $\mathrm{F} 2_{\mathrm{HN}}$ able to confer sequence specificity to the $\mathrm{F} 1-\mathrm{F} 2_{\mathrm{HN}}-\mathrm{F} 2$ protein and yet apparently be unable to bind to RNA in isolation? We hypothesized that the answer might lie in the changes in conformational entropy that take place when a complex is formed. For $\mathrm{F}_{\mathrm{HN}}$ alone, it is only the interactions made by the domain with RNA that can offset the considerable entropic penalty of binding, and it appears that they are insufficient to do so to the level that we might observe an interaction. In the context of $\mathrm{F} 1-\mathrm{F} 2_{\mathrm{HN}}-\mathrm{F} 2$, however, $\mathrm{F} 2{ }_{\mathrm{HN}}$ is anchored on both sides by ZFs that also make interactions with the RNA, thereby giving $\mathrm{F} 2_{\mathrm{HN}}$ the "opportunity" to make sequencespecific interactions with its GCC target sequence without having to pay such a large entropic price.

On the basis of this hypothesis, we predicted that a three$\mathrm{ZF}$ protein with the order of the domains switched (F1-F2$\mathrm{F} 2_{\mathrm{HN}}$ ) would be less discriminating in its RNA-binding activity, because the $\mathrm{F} 2{ }_{\mathrm{HN}}$ domain was only "guided" on one side (Figure $5 \mathrm{~A}$ ). We therefore expressed and purified F1-F2$\mathrm{F} 2_{\mathrm{HN}}$ and measured its affinity for GGUGGUGCC and GGUGGUGGU by ITC (Figure 5B). Consistent with our hypothesis, the ability of this protein to distinguish the two RNAs was diminished $\left(K_{\mathrm{a}}=1.01 \pm 0.11\right.$ and $0.34 \pm 0.04 \times$ $10^{6} \mathrm{M}^{-1}$, respectively-a threefold discrimination power rather than the fivefold observed for $\mathrm{F} 1-\mathrm{F} 2{ }_{\mathrm{HN}}-\mathrm{F} 2$ ). It is also noticeable that the affinity of $\mathrm{F} 1-\mathrm{F} 2-\mathrm{F} 2{ }_{\mathrm{HN}}$ for GGUGGUGCC is significantly lower than that of $\mathrm{F} 1-\mathrm{F} 22_{\mathrm{HN}}-\mathrm{F} 2$ for GGUGCCGGU. This observation suggests that both the sequence-discrimination properties and the binding efficiency of $\mathrm{F} 2_{\mathrm{HN}}$ are position-dependent. Currently, however, we do not understand the basis for this change in affinity, although we speculate that it might also be related to changes in conformational entropy that occur upon binding.

We have created a combinatorial library of RanBP2-type ZF domains in an effort to select variants with distinct RNAbinding preferences. Our data show that the $\mathrm{F} 2_{\mathrm{HN}}$ variant can
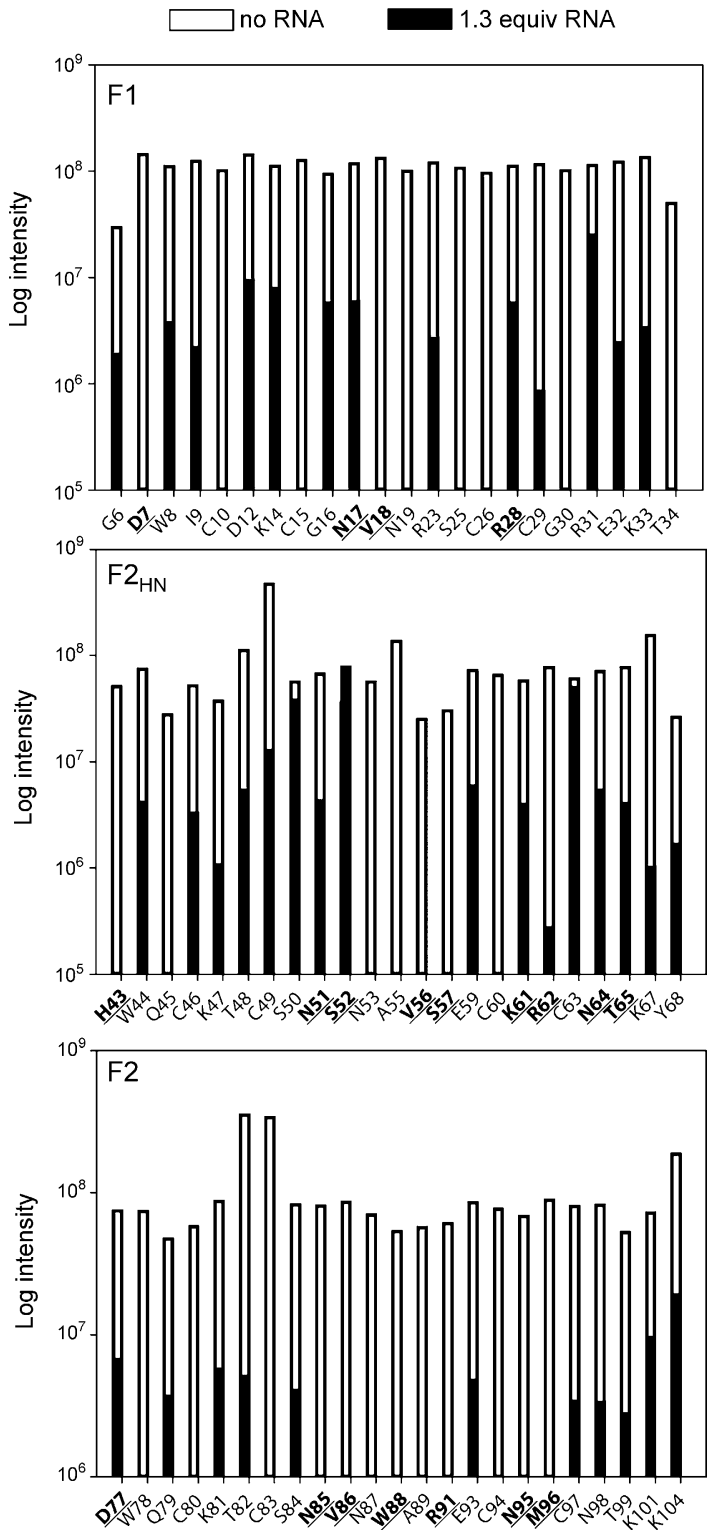

Figure 4. Intensity changes from a ${ }^{15} \mathrm{~N}-\mathrm{HSQC}$ titration in which GGUGCCGGU was added stepwise into ${ }^{15} \mathrm{~N}-\mathrm{F} 1-\mathrm{F} 2_{\mathrm{HN}}-\mathrm{F} 2$. The bars within a group correspond to the integrated intensities measured in the absence (white) and presence of 1.3 molar equivalents of RNA (black). The integrated signal intensity was measured for each residue of $\mathrm{F} 1$ (top), $\mathrm{F} 2 \mathrm{HN}_{\mathrm{HN}}$ (middle), and F2 (bottom). Amino acids predicted to contact RNA on the basis of the crystal structure or showed significant changes in the NMR chemical shifts in published titration experiments ${ }^{[19]}$ are indicated in bold and underlined.

successfully discriminate the sequence GCC over GGU and AAA. However, this discrimination is only observed in the context of a three-ZF polypeptide, and negligible binding of $\mathrm{F} 2_{\mathrm{HN}}$ alone to RNA is observed. On the other hand, we were able to select variants (such as $\mathrm{F} 2_{\mathrm{VA}}$ and $\mathrm{F} 2_{\mathrm{YT}}$ ) with higher affinities for RNA, but which lost their ability to discriminate different target sequences. These data highlight the trade-off between specificity and affinity that is not only at the heart of protein engineering efforts of this type but also a cornerstone of biology. We hypothesise that other classes of proteins (and 
A)
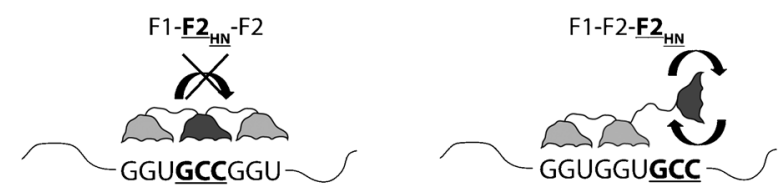

B)
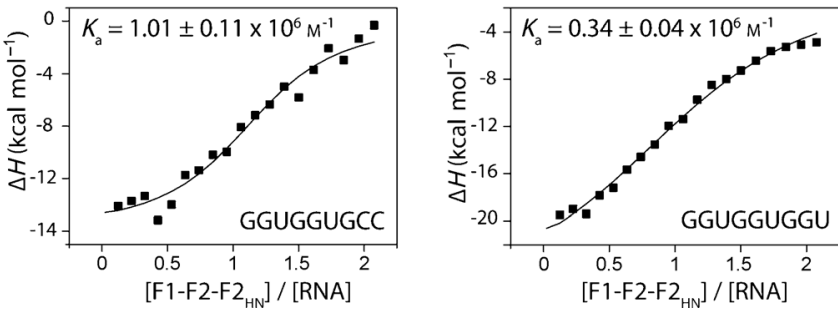

Figure 5. A) Schematic representation of the two three-ZF proteins containing $\mathrm{F}_{\mathrm{HN}}$ : $\mathrm{F} 1-\mathrm{F} 2_{\mathrm{HN}}-\mathrm{F} 2$, and $\mathrm{F} 1-\mathrm{F} 2-\mathrm{F} 2_{\mathrm{HN}}$. $\mathrm{F} 2 \mathrm{HN}$ will display less conformational freedom when in the central position of the $3-\mathrm{ZF}$ protein. B) ITC data for the interaction of F1-F2-F2 ${ }_{\mathrm{HN}}$ with GGUGGUGCC (left) and GGUGGUGGU (right). The fitted $K_{\mathrm{a}}$ values and the standard error derived from two independent titrations are shown.

other molecules) that use modular domains connected by flexible linkers to bind a target molecule will display similar properties. That is, domains positioned at the end of a modular array will display less selectivity than internally positioned modules. This idea has broad implications across biology and drug design. To our knowledge, these are some of the first data to demonstrate that domain ordering dictates the discrimination ability of modules in a multidomain protein. Although on one hand it might appear that the affinity of the $\mathrm{F} 2_{\mathrm{HN}}$ domain is therefore too low to be useful, it is clear that the domain imparts sequence specificity. It is worth noting that DNA-binding classical ZF domains, one of the greatest success stories of protein engineering, ${ }^{[26]}$ have never been demonstrated to bind to DNA as individual domains; they are employed only as 3-6 domain proteins, with each domain providing specificity but only a small contribution to affinity. $\mathrm{F} 2_{\mathrm{HN}}$ represents an important milestone in the design of sequence-specific RNA-binding modules based on ZF domains, as proof of principle that the specificity of these domains can be successfully altered.

Received: March 4, 2014

Keywords: combinatorial chemistry - phage display . protein design $\cdot$ RNA binding $\cdot$ zinc fingers

[1] J. S. Mattick, Bioessays 2003, 25, 930.

[2] B. Maher, Nature 2012, 489, 46.

[3] L. Chen, J. M. Tovar-Corona, A. O. Urrutia, Int. J. Evol. Biol. 2012, 596274.
[4] M. Krawczak, J. Reiss, D. N. Cooper, Hum. Genet. 1992, 90, 41.

[5] a) N. Shiga, Y. Takeshima, H. Sakamoto, K. Inoue, Y. Yokota, M. Yokoyama, M. Matsuo, J. Clin. Invest. 1997, 100, 2204; b) A. Nakamura, S. Takeda, Neuropathology 2009, 29, 494.

[6] S. Mazoyer, N. Puget, L. Perrin-Vidoz, H. T. Lynch, O. M. Serova-Sinilnikova, G. M. Lenoir, Am. J. Hum. Genet. 1998, 62, 713.

[7] H. C. Dietz, D. Valle, C. A. Francomano, R. J. Kendzior, Jr., R. E. Pyeritz, G. R. Cutting, Science 1993, 259, 680.

[8] C. Mayr, D. P. Bartel, Cell 2009, 138, 673.

[9] J. Y. Park, W. Li, D. Zheng, P. Zhai, Y. Zhao, T. Matsuda, S. F. Vatner, J. Sadoshima, B. Tian, PLoS One 2011, 6, e22391.

[10] Y. M. Loo, M. Gale, Jr., Immunity 2011, 34, 680.

[11] X. Wang, J. McLachlan, P. D. Zamore, T. M. Hall, Cell 2002, 110, 501.

[12] S. Dong, Y. Wang, C. Cassidy-Amstutz, G. Lu, R. Bigler, M. R. Jezyk, C. Li, T. M. Hall, Z. Wang, J. Biol. Chem. 2011, 286, 26732.

[13] a) R. O. Emerson, J. H. Thomas, PLoS Genet. 2009, 5, e1000325; b) J. Tilsner, O. Linnik, N. M. Christensen, K. Bell, I. M. Roberts, C. Lacomme, K. J. Oparka, Plant J. 2009, 57, 758; c) Y. Wang, C. G. Cheong, T. M. Hall, Z. Wang, Nat. Methods 2009, 6, 825; d) R. Choudhury, Y. S. Tsai, D. Dominguez, Y. Wang, Z. Wang, Nat. Commun. 2012, 3, 1147; e) A. Cooke, A. Prigge, L. Opperman, M. Wickens, Proc. Natl. Acad. Sci. USA 2011, 108, 15870.

[14] a) Y. Wang, L. Opperman, M. Wickens, T. M. Hall, Proc. Natl. Acad. Sci. USA 2009, 106, 20186; b) M. T. Miller, J. J. Higgin, T. M. Hall, Nat. Struct. Mol. Biol. 2008, 15, 397; c) Y. K. Gupta, D. T. Nair, R. P. Wharton, A. K. Aggarwal, Structure 2008, 16, 549; d) G. Lu, T. M. Hall, Structure 2011, 19, 361.

[15] Z. T. Campbell, E. Menichelli, K. Friend, J. Wu, J. Kimble, J. R. Williamson, M. Wickens, J. Biol. Chem. 2012, 287, 18854.

[16] K. Friend, Z. T. Campbell, A. Cooke, P. Kroll-Conner, M. P. Wickens, J. Kimble, Nat. Struct. Mol. Biol. 2012, 19, 176.

[17] Z. T. Campbell, D. Bhimsaria, C. T. Valley, J. A. RodriguezMartinez, E. Menichelli, J. R. Williamson, A. Z. Ansari, M. Wickens, Cell Rep. 2012, 1, 570.

[18] M. R. O’Connell, M. Vandevenne, C. D. Nguyen, J. M. Matthews, R. Gamsjaeger, D. J. Segal, J. P. Mackay, Angew. Chem. 2012, 124, 5467; Angew. Chem. Int. Ed. 2012, 51, 5371.

[19] F. E. Loughlin, R. E. Mansfield, P. M. Vaz, A. P. McGrath, S. Setiyaputra, R. Gamsjaeger, E. S. Chen, B. J. Morris, J. M. Guss, J. P. Mackay, Proc. Natl. Acad. Sci. USA 2009, 106, 5581.

[20] a) A. Klug, Annu Rev Biochem 2010, 79, 213; b) D. Carroll, Genetics 2011, 188, 773.

[21] C. D. Nguyen, R. E. Mansfield, W. Leung, P. M. Vaz, F. E. Loughlin, R. P. Grant, J. P. Mackay, J. Mol. Biol. 2011, 407, 273.

[22] B. Farina, R. Fattorusso, M. Pellecchia, ChemBioChem 2011, 12, 2837.

[23] F. E. Loughlin, M. Lee, J. M. Guss, J. P. Mackay, Acta Crystallogr. Sect. F 2008, 64, 1175.

[24] J. F. Meckler, M. S. Bhakta, M. S. Kim, R. Ovadia, C. H. Habrian, A. Zykovich, A. Yu, S. H. Lockwood, R. Morbitzer, J. Elsaesser, T. Lahaye, D. J. Segal, E. P. Baldwin, Nucleic Acids Res. 2013, 41, 4118.

[25] M. Jerabek-Willemsen, C. J. Wienken, D. Braun, P. Baaske, S. Duhr, Assay Drug Dev. Technol. 2011, 9, 342.

[26] H. Pearson, Nature 2008, 455, 160. 